\title{
Diversidade e alteridade no cuidado em saúde mental: marcadores e costuras para o trânsito da pandemia
}

\author{
Fabio Scorsolini-Comin ${ }^{1}$
}

(D) https://orcid.org/0000-0001-6281-3371

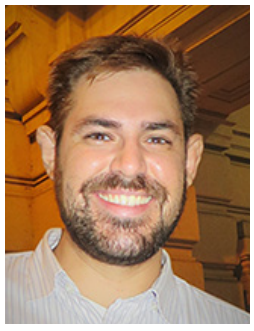

A suspensão de certezas outrora produzidas, em um cenário de caos e instabilidade, marcou o ano de 2020. Ainda que estejamos atravessando uma tormenta global, não sendo possível ainda tratarmos de um cenário pós-pandemia, o conhecimento científico reafirmase como um norte, reforçando o papel dos periódicos no sentido de veicularem produções relevantes e que passaram por um denso processo de avaliação para se apresentarem à comunidade como evidências e apontamentos para a prática(1). Na área de saúde mental, tão debatida e polemizada durante a pandemia(2-3), esse desafio tem nos encorajado no sentido de, coerentes com a missão que aviva a SMAD, fortalecermos nosso processo editorial e nosso compromisso ético, político e científico diante das diversas intempéries.

O primeiro fascículo do ano de 2021 da SMAD é aberto com um editorial bastante oportuno em tempos nos quais se discute sobre a necessidade de respeitarmos, acolhermos e cuidarmos em meio às diversidades e aos múltiplos marcadores sociais da diferença. De autoria de Christoph Käppler, da Universidade de Dortmund, na Alemanha, e de Fabiana Faleiros Santana Castro, da Universidade de São Paulo, este editorial acena para a necessidade de refletir sobre uma perspectiva centrada na pessoa no contexto da reabilitação, ou seja, considerando elementos humanistas que ultrapassam a noção de autonomia e nos convidam a pensar a autenticidade e a congruência, condições fundamentais para um cuidado que se deseja integral.

O primeiro artigo que compõe este fascículo é intitulado "Binge drinking e fatores associados em indígenas da etnia Karipuna", da autoria de Fernanda Matos Fernandes Castelo-Branco, da Universidade Federal do Amapá, e Divane de Vargas, da Universidade de São Paulo. O binge drinking emerge como um fenômeno a ser compreendido junto a 230 indígenas Karipuna no território do Oiapoque. O estudo apontou para a necessidade de se conhecer as especificidades dessa população, a fim de que possam ser construídas estratégias educativas e de prevenção que considerem os contextos desenvolvimentais dessas comunidades, evitando a adoção de ações em saúde que sejam apenas importadas de cenários que não guardam relações com o modo como o álcool é significado nas comunidades pesquisadas.

\footnotetext{
${ }^{1}$ Universidade de São Paulo, Escola de Enfermagem de Ribeirão Preto, Centro Colaborador de la OPS/OMS para el Desarrollo
} de la Investigación en Enfermería, Ribeirão Preto, SP, Brasil.

\section{Como citar este artigo}

Scorsolini-Comin F. Diversity and otherness in mental health care: markers and seams for the transit of the pandemic. SMAD, RevEletrônica SaúdeMentalÁlcoolDrog.2021;17(1):1-4.doi: https://dx.doi.org/10.11606/issn.1806-6976.smad.2021.000175. 
O segundo artigo, "Facilidades de adesão familiar no tratamento da dependência química: percepção dos familiares", da autoria de Ana Carolina Belmonte Assalin, Sonia Regina Zerbetto, Bianca Oliveira Ruiz, Priscila Souza Cugler e Sarah Salvador Pereira, foi desenvolvido em um Centro de Atenção Psicossocial - álcool e drogas do interior do estado de São Paulo. Segundo as autoras do estudo, reconhecer os aspectos que potencializam a adesão da família é fundamental para que o cuidado em saúde nesses equipamentos acolha de modo mais propositivo esses familiares e suas necessidades, produzindo melhores desfechos no cuidado ofertado.

A construção do matriciamento é discutida no estudo "Matriciamento: uma experiência ambulatorial", escrito por Marina Bianco Perrone e Thiago Marques Fidalgo, da Universidade Federal de São Paulo. A pesquisa-ação desenvolvida ao longo de sete anos por uma terapeuta ocupacional apontou para a importância do trabalho matriciado, permitindo incrementos importantes para a construção clínica. Os autores recomendam a construção coletiva de projetos terapêuticos que possam convocar diferentes audiências e inteligibilidades no processo de cuidar.

Pesquisadoras e pesquisadores da Faculdade de Medicina de Marilia, da Universidade do Oeste Paulista e da Universidade Estadual Paulista "Julio de Mesquita Filho" apresentam o artigo "Condições de vida e saúde de idosos com transtornos mentais de acordo com o sexo". Neste estudo, Maria Cássia Corrêa Mazzi Freire, Maria José Sanches Marin, Carlos Alberto Lazarini e Daniela Garcia Damaceno partem de dados produzidos em um ambulatório de saúde mental. Corroborando achados descritos na literatura, as mulheres buscam o cuidado nesse equipamento de modo mais intenso, ao contrário do que pode ser observado nos homens. Para estes, isso repercute, por exemplo, em diagnósticos mais tardios. As estratégias devem considerar o gênero como um marcador importante, a fim de que as ações possam, de fato, atingir seus objetivos junto a esses idosos.

Na sequência, o artigo "Nutrizes usuárias de drogas e o desfecho da amamentação: estudo de coorte", de Silmara de Fátima Teixeira Ribeiro e Rosa Aurea Quintella Fernandes, da Universidade Guarulhos, busca conhecer o desfecho do aleitamento materno de mães usuárias de drogas lícitas e ilícitas. O estudo realizado com 108 mulheres concluiu que as mães usuárias de drogas praticaram o aleitamento exclusivo, mesmo aquelas que consumiam maconha e cocaína de modo abusivo. Em que pese a consideração de que o consumo de drogas representa um fator de risco para o desmame precoce, o perfil dessas usuárias pode apontar para a construção de diferentes estratégias para a mudança do comportamento materno durante o período da amamentação.

Pesquisa realizada a partir de uma parceria entre a Santa Casa de Misericórdia de Presidente Prudente e a Faculdade de Medicina de Marília teve como objetivo caracterizar os idosos atendidos em um Serviço de Urgência e Emergência Psiquiátrica quanto ao ser perfil sociodemográfico e associação com o diagnóstico psiquiátrico e a prescrição de medicamentos. Este estudo, intitulado "Idosos atendidos em um Serviço de Urgência e Emergência Psiquiátrica", escrito por Mayara Giuli dos Santos Cordeiro e colaboradores, apontou que os diagnósticos prevalentes entre 2015 e 2016 foram os de esquizofrenia e os transtornos mentais e comportamentais decorrentes do uso de substâncias psicoativas. Em termos das práticas profissionais discutidas pelas autoras e autores, destaca-se a manutenção de um modelo de atendimento que ainda se contrapõe às recomendações do Ministério da Saúde para uma reabilitação psicossocial.

O estudo em sequência é intitulado "Grau de dependência à nicotina de pacientes atendidos para tratamento do tabagismo em universidade pública", de Rafael da Rosa Maciel, Lidia Dalgallo, Erildo Vicente Müller e Elaine Cristina Antunes Rinaldi, da Universidade Estadual de Ponta Grossa. Os participantes desta investigação foram pacientes atendidos ao longo de quatro anos em um projeto de extensão universitária sobre o tabagismo. Os dados apontaram que o grau de dependência química à nicotina esteve associado à dependência física e psicológica. Esse achado evidencia a necessidade de que as estratégias de educação em saúde devem envolver uma escuta para os diferentes elementos envolvidos no tabagismo, com destaque para os recursos produzidos por meio da associação de terapias medicamentosas a uma abordagem cognitivo-comportamental em saúde.

A humanização no cuidado em saúde mental é abordada por Deivson Wendell da Costa Lima e Lívia Dayane Sousa Azevedo, da Universidade de São Paulo, em parceria com pesquisadores da Universidade do Estado do Rio Grande do Norte: Ana Karla Ramalho Paixão, Kelianny Pinheiro Bezerra, Rodrigo Jácob Moreira de Freitas e Fátima Raquel Rosado. O estudo priorizou as inteligibilidades produzidas por enfermeiros atuantes em um hospital psiquiátrico do interior do nordeste brasileiro, revelando a associação do cuidado humanizado com o modelo manicomial, promovendo ações desarticuladas e que não consideram a participação do paciente, em um movimento que ainda revela a soberania do discurso biomédico, hospitalocêntrico e médico-centrado no campo da saúde mental.

O artigo "A percepção dos trabalhadores de saúde mental sobre a atuação do redutor de danos", da autoria de Diego Fernandes Leal, Vanessa Alvez Mora da Silva, Luana Ribeiro Borges, Sandra Beatris Diniz Ebling, da Universidade Federal do Pampa, e de Aline Ost dos Santos, da Secretaria Municipal de Saúde de Uruguaiana, no Rio Grande do Sul, teve como contexto de investigação um Centro de Atenção Psicossocial de Álcool e outras drogas da região. O 
grupo focal narrativo apontou para o fato de que o redutor de danos, no serviço em tela, possui a capacidade de criar e sustentar vínculos para a realização de um acolhimento efetivo e de qualidade. Esse dado corrobora o movimento permanente em direção à desconstrução do estigma do usuário de substâncias em sociedade, reforçando a adequação dessa proposta de cuidado em saúde mental.

O estudo "Treinamento em transtornos mentais comuns na enfermaria: uso de metodologias ativas na construção do cuidado", de Marília Girão de Oliveira Machado, da Universidade Federal do Ceará, e de Cynthia Lima Sampaio, do Hospital Universitário Walter Cantídio, de Fortaleza, buscou conhecer as percepções de enfermeiras que atuam em leitos psiquiátricos de um hospital geral sobre o emprego de metodologias ativas em treinamentos no campo da saúde mental. Tais metodologias foram reconhecidas como positivas e potentes nesse contexto, associando-se à possibilidade de uma aprendizagem mais significativa e que permita o engajamento dos profissionais.

Os impactos do alcoolismo nas relações familiares vêm sendo estudados na literatura a partir de diferentes perspectivas e na visão de diferentes atores envolvidos nesse processo. O estudo produzido por Ketlen Patrycia Alves Cordeiro, Lavinia Luzia Gomes Souza, Raquel Schwenck de Mello Vianna Soares, Ludmila Cotrim Fagundes e Wellington Danilo Soares, ligados à Faculdade de Saúde e Humanidades Ibituruna, à Universidade Federal dos Vales do Jequitinhonha e Mucuri e à Universidade Estadual de Montes Claros, destacou diferentes sentidos sobre o alcoolismo produzido por homens que frequentam os Alcóolicos Anônimos. Entre esses sentidos destacam-se aqueles relacionados ao início do uso abusivo, às consequências para a família e às estratégias de ajuda. Entre essas estratégias apresentam-se tanto a família como o apoio de entidades como os Alcóolicos Anônimos.

Thiago Guimarães Da-Silva, Pedro Fonseca De Vasconcelos e Ivan Gilson Silva Moura, da Faculdade de Saúde Santo Agostinho, em Vitória da Conquista, Bahia, apresentam o estudo intitulado "Uma abordagem atual da utilização de antidepressivos no manejo da depressão pós-parto". A partir de uma revisão integrativa de literatura, apontou-se a sertralina como fármaco predominante no tratamento farmacológico da depressão puerperal. A revisão também salientou que não há consenso sobre a superioridade da terapia farmacológica em detrimento às psicoterapias para o tratamento de depressão pós-parto.

O último estudo que compõe o presente fascículo destaca a temática da saúde mental em estudantes universitários, conteúdo este que vem sendo bastante investigado na contemporaneidade, o que foi acompanhado pelas discussões oportunizadas ao longo de todo o ano de 2020, no contexto da pandemia do novo coronavírus e da COVID-19(4-6). Para contribuir com essa discussão, Larissa Barreto dos Santos, Karina Gomes do Nascimento, Andréia Guedes Oliva Fernandes e Talita de Cássia Raminelli-da-Silva, do Centro Universitário Euro-Americano, apresentaram o artigo intitulado "Prevalência, severidade e fatores associados à depressão em estudantes universitários", que identificou a sintomatologia depressiva em 521 estudantes universitários do Distrito Federal. Na presente investigação a renda familiar e o semestre cursado foram fatores associados com a severidade da depressão, de modo que essas variáveis devem ser melhor compreendidas em estudos vindouros para compor um rol de fatores de risco e de proteção que podem ser elencados no contexto do cuidado em saúde mental voltado aos universitários.

Finalizamos esta apresentação com o desejo de que esses estudos do primeiro fascículo de 2021 possam ser apreciados em termos das realidades retratadas e dos desafios em saúde mental assinalados por esses contextos, o que deve fomentar constantemente a busca pelo reconhecimento de recursos e pela proposição de estratégias que façam frente a esses circunscritores. Essas produções devem ser revisitadas pelos leitores a partir de diferentes perspectivas e também por meio do convite transformador corporificado na pandemia da COVID-19. Este cenário - em trânsito - deve nos possibilitar a construção de ações que dialoguem tanto com a intermitência dessa condição quanto das possibilidades de leitura que nos afetarão ainda por um longo tempo. Que em 2021 possamos assumir esses desafios amparados pelas aprendizagens de 2020 e pela esperança sempre renovada quando pensamos no cuidado em saúde mental, essa atenção que não pode ser desenvolvida sem uma abertura suficiente para a diversidade, anunciada desde o editorial, e também para a alteridade, o que nos obriga a olhar, a considerar e acolher o(s) outro(s) para além dos nossos alcances de pesquisa, ensino e prática.

\section{Referências}

1. Costa ICP, Sampaio RS, Souza FAC, Dias TKC, Costa BHS, Chaves ECL. Produção científica em periódios online sobre o novo coronavírus (COVID-19): pesquisa bibliométrica. Texto Contexto Enferm. 2020; 29:e20200235. doi: https://doi.org/10.1590/1980-265x-tce-2020-0235.

2. Ornell F, Schuch JB, Sordi AO, Kessler FHP. "Pandemic fear"' and COVID-19: Mental health burden and strategies. Braz J Psychiatry. 2020;42(3):232-5. doi: https://doi.org/10.1590/1516-4446-2020-0008 
3. Wang C, Pan R, Wan X, Tan Y, Xu L, Ho CS, Ho RC. Immediate psychological responses and associated factors during the initial stage of the 2019 coronavirus disease (COVID-19) epidemic among the general population in China. Int J Environ Res Public Health. 2020;17(5):1729. doi: https://doi.org/10.3390/ijerph17051729

4. Gomes CFM, Pereira Junior RJ, Cardoso JV, Silva DA. Transtornos mentais comuns em estudantes universitários: abordagem epidemiológica sobre vulnerabilidades. SMAD, Rev Eletron Saúde Mental Álcool Drog. 2020;16(1):1-8. doi: https://doi.org/10.11606/issn.1806-6976.smad.2020.157317

5. Savitsky B, Findling Y, Ereli A, Hendel T. Anxiety and coping strategies among nursing students during the covid-19 pandemic. Nurse Educ Pract. 2020;46:102809. doi: https://doi.org/10.1016/j.nepr.2020.102809

6. Patias ND, Hohendorff JV, Cozzer AJ, Flores PA, Scorsolini-Comin F. Mental health and coping strategies in undergraduate and graduate students during COVID-19 pandemic. Trends in Psychol. 2021;29(1). doi: https://doi. org/10.1007/s43076-021-00069-z 